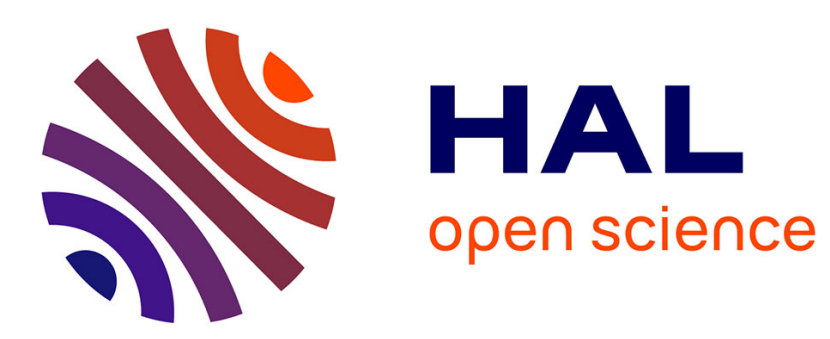

\title{
Flow-induced vibrations of high mass ratio flexible filaments freely hanging in a flow
}

Lionel Schouveiler, Christophe Eloy, Patrice Le Gal

\section{To cite this version:}

Lionel Schouveiler, Christophe Eloy, Patrice Le Gal. Flow-induced vibrations of high mass ratio flexible filaments freely hanging in a flow. Physics of Fluids, 2005, 17, pp.Art. 047104. 10.1063/1.1878292 . hal-00014491

\section{HAL Id: hal-00014491 \\ https://hal.science/hal-00014491}

Submitted on 28 Nov 2005

HAL is a multi-disciplinary open access archive for the deposit and dissemination of scientific research documents, whether they are published or not. The documents may come from teaching and research institutions in France or abroad, or from public or private research centers.
L'archive ouverte pluridisciplinaire HAL, est destinée au dépôt et à la diffusion de documents scientifiques de niveau recherche, publiés ou non, émanant des établissements d'enseignement et de recherche français ou étrangers, des laboratoires publics ou privés. 


\title{
FLOW-INDUCED VIBRATIONS OF HIGH MASS RATIO FLEXIBLE FILAMENTS FREELY HANGING IN A FLOW
}

\author{
Lionel Schouveiler, Christophe Eloy, Patrice Le Gal
}

Institut de Recherche sur les Phénomènes Hors Equilibre

UMR 6594 CNRS - Universités d'Aix-Marseille

Technopôle de Château-Gombert

49, rue Frédéric Joliot Curie, BP 146

F-13384 Marseille Cedex 13, France

\begin{abstract}
The behavior of high mass ratio flexible filaments freely hanging in steady horizontal uniform flows is experimentally and theoretically investigated. When the flow velocity is small, static equilibrium states, where the filaments are inclined to the flow, are observed. Then, above a critical value of the wind velocity, the filaments exhibit periodic oscillations in the vertical plane. The problem is theoretically addressed considering the beam theory equations for the filament dynamics where the action of the flowing fluid is modeled using semi-empirical expressions. These equations are first solved for the stationary equilibrium states. Then, the stability of these steady solutions relatively to small perturbations is analyzed. A good agreement between experimental and theoretical results is found.
\end{abstract}




\section{INTRODUCTION}

Interactions of flows with flexible elongated structures are encountered in many industrial fields such as ocean engineering (towing cables, mooring lines, risers) or civil engineering (cables of suspension bridges or of hanging roofs). Moreover, because they can induce structure vibrations that are a potential cause of damage, they have received considerable attention. Flow-induced vibrations can arise from different mechanisms which were classified by Blevins ${ }^{1}$. For steady flows, efforts have mainly concerned the vortexinduced vibrations (referred to, hereafter, as VIV) and comprehensive reviews were published by Bearman ${ }^{2}$ and more recently by Williamson and Govardhan ${ }^{3}$. Most of the studies devoted to flow-induced vibrations of elongated bodies are motivated by ocean engineering applications for which the mass ratio $\mu$ is usually less than 15. This parameter $\mu$ is defined as the ratio of the "oscillating mass", including the mass of the structure and the added fluid mass, divided by the displaced fluid mass. The mass ratio $\mu$ is the primary parameter of VIV (e.g. Ref. 2): the lower $\mu$, the higher the susceptibility of structures to VIV.

On contrary, in the present study, we consider flexible filaments freely hanging in a wind. This configuration leads to a high value of $\mu$ of the order of 1000 . Note that similar investigations have recently been reported (Facchinetti et al. ${ }^{4}$ ) for a mass ratio $\mu$ slightly above 2. Comparison with our results will emphasize the strong influence of $\mu$ on the filament dynamics.

Finally, we should also mention the extensive work of Païdoussis and co-workers, whose references can be found in the recent article of Païdoussis et $a l^{5}$, devoted to the stability of a flexible cylinder in an axial flow, that is with an initial incidence angle equal to zero. The theoretical analysis reported in the present paper generalizes these studies to the case of a flexible cylinder inclined to the flow, i.e. for arbitrary angles of incidence.

In the following section (§II.), details on the experimental setup and on our experimental observations are presented. In Section III., we derive the governing equations describing the filament equilibrium and dynamics. A theoretical linear stability analysis is also performed whose results are compared with experiments. Finally, brief complementary discussions are given in Section IV together with the conclusion. 


\section{EXPERIMENTS}

\section{A. Experimental setup}

Our experiments are performed in a low turbulence wind tunnel. Its horizontal working section is $1 \mathrm{~m}$ long and has a square cross-section of $0.5 \times 0.5 \mathrm{~m}^{2}$. The free stream velocity $U$ is measured with a conventional Pitot tube and can be continuously varied up to $25 \mathrm{~m} \mathrm{~s}^{-1}$. The quality of the tunnel has been quantified, prior to this study, with a constant temperature hot wire anemometer. At $25 \mathrm{~m} \mathrm{~s}^{-1}$ the free stream uniformity was better than $0.5 \%$ over $80 \%$ of the tunnel width and the turbulence level measured on the tunnel axis was close to $0.1 \%$. The filaments are made of silicone and have a circular and constant cross-section $A=\pi d^{2} / 4$ and a variable length $l$.

To be maintained in the wind, each filament is inserted in a steel tube having the same inner diameter $d$. This support is fixed outside of the test section and crosses perpendicularly the wind tunnel top wall. It is bent at right angle in such a way that its final part is parallel to the free stream, this part is $10 \mathrm{~cm}$ long. The filament freely hangs at this tube end. This setup assures a clamped boundary condition at the upstream end with the filament parallel to the free stream, the other filament end being free.

For the present study, visualizations of the filament are performed through the transparent side walls of the wind tunnel test section. Images are captured with a video camera operating at $60 \mathrm{~Hz}$ and then processed to get quantitative results. For instance, the flapping frequencies of the filament are obtained by a FFT analysis of space-time images.

All the filaments are made of silicone with a Young's modulus $E=2.5 \mathrm{MPa}$. Their flexural rigidity is $E I$ where $I=\pi d^{4} / 64$. Extensive experiments are carried out for filaments of diameters $d=1.2$ and $1.8 \mathrm{~mm}$, but some measurements have been validated with filaments of diameter $d=1.6 \mathrm{~mm}$. The length $l$ of these filaments is varied up to about $30 \mathrm{~cm}$. The density $\rho$ of silicone is about $950 \mathrm{~kg} \mathrm{~m}^{-3}$ and experiments are performed at a temperature close to $20^{\circ} \mathrm{C}$ in such a way that the values $v_{f l}=15 \times 10^{-6} \mathrm{~m}^{2} \mathrm{~s}^{-1}$ and $\rho_{f l}=1.2 \mathrm{~kg} \mathrm{~m}^{-3}$ are considered for the kinematical viscosity and density of the flowing air, respectively. This results in a mass ratio $\mu$ of about 800 . Note that for a non-confined cylinder the added fluid mass is equal to 
the displaced fluid mass (see the detailed analysis of Lopes et al. ${ }^{6}$ ) in such a way that the mass ratio simply writes $\mu=\left(\rho+\rho_{f l}\right) / \rho_{f l}$.

\section{B. Experimental observations}

The first experiments consist in gradually increasing the free stream velocity $U$ for a given filament (i.e. for given $d$ and $l$ ). For low wind velocities, the filament appears to be stationary. Then, when $U$ exceeds a well-defined critical value $U_{c}$, it exhibits a flapping motion. A visual inspection reveals that this motion takes place in a vertical plane, at least for the wind velocities considered here.

The static equilibrium positions mainly correspond to the balance between the gravity force and the fluid load. Therefore they depend on the flow velocity $U$. Figure 1 presents superimposed views of steady positions of a filament of diameter $d=1.2 \mathrm{~mm}$, length $l=12 \mathrm{~cm}$ and for ten values of $U$ ranging from 0 to $13.6 \mathrm{~m} \mathrm{~s}^{-1}$. For this particular filament, we find $U_{c}=13.7 \mathrm{~m} \mathrm{~s}^{-1}$. For all these static equilibria, the filament axis is totally contained in a vertical plane (parallel to gravity and free stream). As $U$ is increased, the filament tends to align with the free stream, so its incidence angle decreases. Moreover, images of Fig. 1 show that except close to the clamped end, the filament is nearly rectilinear.

Unsteady behavior of the filament spontaneously takes place when the free stream velocity $U$ exceeds a threshold $U_{c}$. Critical values $U_{c}$ are determined for numerous combinations of $d$ and $l$ and are reported on Fig. 2 for $d=1.2 \mathrm{~mm}$ and $1.8 \mathrm{~mm}$. It appears that $U_{c}$ depends on the length $l$ and diameter $d$ of the considered filament. We first note the increase of $U_{c}$ with $d$. Besides, for both diameters, we can observe that the threshold $U_{c}$ strongly decreases with $l$ for short filaments, whereas the dependence of $U_{c}$ on $l$ is weaker for $l$ larger than $15 \mathrm{~cm}$

The unsteady behavior consists in a regular periodic flapping of the filament in a vertical plane i.e. a plane parallel to free stream and gravity. These vertical oscillations are illustrated by Fig. 3 which shows pictures of ten superimposed successive views captured at $60 \mathrm{~Hz}$ during flapping motion. In contrast to short filaments [Fig. 3(a)], one node can be distinguished in the envelope of the flapping motion of long filaments. One of these nodes has been marked by an arrow in Fig. 3(b). But the motion amplitude being quite small, except at the free end, it was not always possible to determine precisely if one or more nodes are present 
along the filaments and consequently to measure accurately the boundary between zero- and one-node flapping.

The flapping of the filament is periodic. In Fig. 4, its frequency $f$ is plotted as a function of $U$ for various filaments. Frequency $f$ is calculated by the spectral analysis of sequences of 360 images captured at $60 \mathrm{~Hz}$. This gives a resolution of about $0.17 \mathrm{~s}^{-1}$. The frequency is found to be constant along the filament. In Fig. 4, it appears that $f$ increases with $U$ and decreases for increasing $d$ or $l$. Note also that plateaus are sometimes observed at threshold. As already mentioned and in contrast to the VIV where vibrations are mainly transverse to the free stream, and perpendicular to the gravity, in the present study strictly vertical, i.e. parallel to the gravity, oscillations are always observed at threshold.

\section{III.THEORETICAL ANALYSIS}

\section{A. Static equilibrium states}

We introduce the curvilinear coordinate $s$ along the filament axis, with $s=0$ at the free end and $s=l$ at the upstream extremity. The incidence angle $\theta(s)$ is the local angle between the free stream and the filament axis. We consider a small filament element ds; forces and moments acting on this small element are displayed in Fig. 5. They consist in a tension $T$ and a shear $Q$ in a cross section, a bending moment $M$, the gravity force and the fluid dynamics load. We consider separately the inviscid force $F$ and the viscous force of components $L$ and $N$. F, $L$ and $N$ are forces per unit length. We assume that a cross section of the straight filament remains plane during all deformations in such a way that the Bernoulli-Euler beam theory can be applied. Moreover, because no notable lengthening is detected during experiments, the center line of the filament is considered as inextensible.

The force balance for static equilibrium writes in the s-wise and transverse direction respectively,

$$
\begin{aligned}
& \frac{\mathrm{d} T}{\mathrm{~d} s}=L-\left(\rho-\rho_{f l}\right) g A \sin \theta, \\
& \frac{\mathrm{d} Q}{\mathrm{~d} s}=F+N-\left(\rho-\rho_{f l}\right) g A \cos \theta .
\end{aligned}
$$

The moment balance gives 


$$
Q=\frac{\mathrm{d} M}{\mathrm{~d} s}=E I \frac{\mathrm{d}^{2} \theta}{\mathrm{d} s^{2}}
$$

where the second equality results from the expression of $M$ given by the Bernoulli-Euler beam theory.

Because the curvature radius of the filament is very large relatively to its diameter $d$, the flow is considered as locally two-dimensional. Therefore, for both components $N$ and $L$ of the viscous force, we use the semi-empirical expressions deduced by Taylor $^{7}$ from force measurements on a smooth circular cylinder set at various incidence angles in a wind:

$$
\begin{aligned}
& N=\frac{1}{2} \rho_{f l} d U^{2}\left(C_{D} \sin ^{2} \theta+4 \operatorname{Re}^{-\frac{1}{2}} \sin ^{\frac{3}{2}} \theta\right), \\
& L=\frac{1}{2} \rho_{f l} d U^{2}\left(5.4 \operatorname{Re}^{-\frac{1}{2}} \cos \theta \sin ^{\frac{1}{2}} \theta\right),
\end{aligned}
$$

where $R e=U d / v_{f l}$ is the Reynolds number and the pressure drag coefficient $C_{D}$ is treated as an adjustable parameter of the model.

The inviscid fluid dynamic force $F$ is equal in magnitude but opposite to the rate of change of the fluid momentum. Using the expression deduced from an elongated-body potential flow theory by Lighthill ${ }^{8}$, we find that

$$
F=-\rho_{f l} A U^{2} \frac{\mathrm{d} \theta}{\mathrm{d} s} \cos ^{2} \theta
$$

where $\rho_{f} A$ is the added mass of fluid per unit length of filament (e.g. Lopes et al. ${ }^{6}$ ). Thus $F$ is proportional to the filament curvature $\mathrm{d} \theta / \mathrm{d} s$. It results that the drag of a straight cylinder in a potential flow is zero, this is known as the d'Alembert's paradox.

At the clamped end the filament is maintained parallel to the wind so that

$$
\theta(s=l)=0 .
$$

Forces and moment acting on the free end cross section are supposed to be negligible giving

$$
\begin{aligned}
& T(s=0)=0, Q(s=0)=0, \\
& M(s=0)=0 \text { i.e. } \frac{\mathrm{d} \theta}{\mathrm{d} s}(s=0)=0 .
\end{aligned}
$$

The above equation system (1-3) together with the force expressions (4-6) and the boundary conditions (7- 
10) are solved numerically using a shooting method with a standard Runge-Kutta integration scheme. The shape $\theta(s)$ of the filament axis is thus deduced as a function of the control parameter $U$. Calculations are repeated for various values of the only free parameter $C_{D}$. Results obtained for the same conditions as that of the experiments of Fig. $1\left(l=12 \mathrm{~cm}\right.$ and $U$ from 0 to $\left.13.6 \mathrm{~m} \mathrm{~s}^{-1}\right)$ and calculated with $C_{D}=0.8$ and 0.9 are plotted in Fig. 6.

Beyond the qualitative agreement revealed by comparing Figs. 1 and 6, a systematic quantitative comparison between the experiments and the theory is performed to determine the suitable value of the drag coefficient $C_{D}$. For this purpose, the incidence angle at the free end $\theta(s=0)$ is used and experimental data are compared to the theoretical results obtained for various values of $C_{D}$. The good agreement between theory and experiments is illustrated by the example in Fig. 7 where $l=12 \mathrm{~cm}, d=1.2 \mathrm{~mm}$ and $C_{D}=0.8$ and 0.9 for the calculations. It allows to validate the Taylor's semi-empirical expressions $(4,5)$ used for the viscous fluid load. Comparisons are repeated for several combinations of $d, l$ and $U$ and the best agreement is found for $C_{D}$ ranging between 0.8 and 0.9. In the following these two values of the drag coefficient will be considered.

\section{B. Linear stability analysis}

By contrast to VIV, the observed flapping motion results directly from an instability and not from a structural response to hydrodynamical forcing. Linear stability analysis of the static equilibrium positions is then performed to determine the theoretical critical values of the free stream velocity and flapping characteristics at, or close to, the threshold.

\section{Governing equation for small displacements}

We first derive the governing equation for the filament element $d s$ and undergoing a small displacement $y(s, t)$ as seen in Fig. 8. $y(s, t)$ is measured from the stationary position defined by $\theta(s)$ the solution of equations (1-3) at a given $U$. According to the experimental observations, only vertical motions are considered. In this case, the normal and tangential components of the fluid velocity relative to the filament are respectively,

$$
v_{N}(s, t)=U \sin \theta+U \cos \theta \frac{\partial y}{\partial s}-\frac{\partial y}{\partial t},
$$




$$
v_{T}(s, t)=-U \cos \theta+\left(U \sin \theta-\frac{\partial y}{\partial t}\right) \frac{\partial y}{\partial s}
$$

The incidence angle $\theta_{i}$ between the free stream and the filament axis is then

$$
\theta_{i}=\theta+\frac{\partial y}{\partial s}-\frac{\cos \theta}{U} \frac{\partial y}{\partial t}
$$

The structural damping of the filament material is neglected here in such a way that the forces and moment acting on the filament element are the same as listed in § III.A (see also Fig. 5). Therefore, to first order in $y$, Newton's second law projected onto the $y$-direction writes

$$
\rho A \frac{\partial^{2} y}{\partial t^{2}}=\frac{\partial}{\partial s}\left(T \frac{\partial y}{\partial s}\right)+F+f+N+n-L \frac{\partial y}{\partial s}-\frac{\partial(Q+q)}{\partial s}-\left(\rho-\rho_{f}\right) g A \cos \theta
$$

and moment balance is

$$
Q+q=\frac{\partial(M+m)}{\partial s}=E I\left(\frac{d^{2} \theta}{d s^{2}}+\frac{\partial^{3} y}{\partial s^{3}}\right)
$$

where upper case letters $T, Q, F, N, L$ and $M$ denote the quantities (functions of $s$ only), to order zero in $y$, given by the static equilibrium equations (1-6). Lower case letters $q, f, n$ and $m$ correspond to the supplementary terms appearing at first order in $y$, and are functions of $s$ and $t$.

Combining the derivative of equation (15) with (2), relation (14) becomes

$$
\rho A \frac{\partial^{2} y}{\partial t^{2}}=\frac{\partial}{\partial s}\left(T \frac{\partial y}{\partial s}\right)+f+n-L \frac{\partial y}{\partial s}-E I \frac{\partial^{4} y}{\partial s^{4}}
$$

The inviscid fluid force is (Lighthill ${ }^{8}$ )

$$
F_{A}=\rho_{f l} A\left(\frac{\partial}{\partial t}-U \cos \theta \frac{\partial}{\partial s}\right) v_{N}
$$

where the normal velocity $v_{N}(s, t)$ is given by (11); hence

$$
f=\rho_{f l} A\left(-\frac{\partial^{2} y}{\partial t^{2}}+2 U \cos \theta \frac{\partial^{2} y}{\partial t \partial s}+U^{2} \cos \theta \sin \theta \frac{d \theta}{d s} \frac{\partial y}{\partial s}-U^{2} \cos ^{2} \theta \frac{\partial^{2} y}{\partial s^{2}}\right)
$$

In equation (16) $n$ is the first order term of expression (4) in which $\theta$ has been replaced by the incidence angle $\theta$ i given by (13) and $U$ by $\left(v_{N}^{2}+v_{T}^{2}\right)^{\frac{1}{2}}$ where the two velocity components are given by $(11,12)$. It results that 


$$
n=\frac{1}{2} \rho_{f l} d U^{2}\left[\left(C_{D} \sin 2 \theta+6 \operatorname{Re}^{-\frac{1}{2}} \cos \theta \sin ^{\frac{1}{2}} \theta\right) \psi+\left(2 C_{D} \sin ^{2} \theta+6 \operatorname{Re}^{-\frac{1}{2}} \sin ^{\frac{1}{2}} \theta\right) \frac{u}{U}\right]
$$

where $\psi=\frac{\partial y}{\partial s}-\frac{\cos \theta}{U} \frac{\partial y}{\partial t}$ and $\frac{u}{U}=-\frac{\sin \theta}{U} \frac{\partial y}{\partial t}$.

An equation similar to (16) has been derived by Païdoussis ${ }^{9,10}$ to describe the small lateral motions of a filament in an axial flow, i.e., small displacements around the static equilibrium state $\theta(s)=0$ for all $s$ between 0 and $l$. In this case, and contrary to the present configuration, the governing equation (16) can be linearized with respect to the (small) incidence angle $\theta$. This situation has been extensively studied and references can be found in the recent series of articles by Païdoussis and co-workers (Païdoussis et al. ${ }^{5}$, Lopes et al. ${ }^{6}$, Semler et al. ${ }^{11}$ ).

Moreover, it should be pointed out that, for the lengths considered here, the filaments in static equilibrium are rectilinear on most of their length, specially close to the flapping threshold (Figs. 1 and 6). Hence, in the following we restrict our stability analysis to a filament having a constant incidence angle equal to the value at free end $\theta(s=0)$. From equations (1) and (5), it results a linear variation of the tension $T$ along the filament.

\section{Results and comparisons}

For the analysis we consider periodic perturbations of the form

$$
y(s, t)=\Re \mathrm{e}\left[Y(s) \mathrm{e}^{\mathrm{i} \omega t}\right],
$$

where $\omega$ is a complex frequency.

Assuming the filament is initially rectilinear (i.e. $\theta$ is constant therefore $T$ is linear in $s$ ) and using the filament length $l$ to make dimensionless the spatial variables (let $\xi=\frac{S}{l}$ and $\eta=\frac{Y}{l}$ ) the governing equation for small displacements (16) becomes

$$
\frac{\mathrm{d}^{4} \eta}{\mathrm{d} \xi^{4}}+a \frac{\mathrm{d}^{2} \eta}{\mathrm{d} \xi^{2}}+b \xi \frac{\mathrm{d}^{2} \eta}{\mathrm{d} \xi^{2}}+\left(c_{1}+i \omega c_{2}\right) \frac{\mathrm{d} \eta}{\mathrm{d} \xi}+\left(\omega^{2} e_{1}+\mathrm{i} \omega e_{2}\right) \eta=0
$$

where the coefficients $a, b, c_{1}, c_{2}, e_{1}$ and $e_{2}$ depend non linearly on $U$ and $\theta$, their expressions are given in the Appendix. A dramatic simplification occurs when $\theta=0$, which corresponds to the classical case of a filament 
in an axial flow (e.g. Païdoussis ${ }^{9,10}$ ). For the situation considered here, the coefficient $c_{1}$ is non zero; this induces a new destabilizing term which effect has never been into account before. The boundary conditions at the clamped end, $\xi=1$, are

$$
\eta=\frac{\mathrm{d} \eta}{\mathrm{d} \xi}=0
$$

and at the free end, $\xi=0$,

$$
\frac{\mathrm{d}^{2} \eta}{\mathrm{d} \xi^{2}}=\frac{\mathrm{d}^{3} \eta}{\mathrm{d} \xi^{3}}=0
$$

To solve the whole governing equation (21) we use a Galerkin method involving the eigenfunctions $\phi_{i}(\xi)$ of the filament without flow. This is applicable because we assume that the fluid load is negligible at the free end in such a way that the boundary conditions $(22,23)$ are identical with or without flow. Then, the method consist to approximate the filament displacement $\eta(\xi)$ by the truncated series

$$
\eta(\xi)=\sum_{i=1}^{n} a_{i} \phi_{i}(\xi)
$$

where

$$
\phi_{i}(\xi)=\frac{\operatorname{ch} k_{i}(1-\xi)-\cos k_{i}(1-\xi)}{\operatorname{ch} k_{i}+\cos k_{i}}-\frac{\operatorname{sh} k_{i}(1-\xi)-\sin k_{i}(1-\xi)}{\operatorname{sh} k_{i}+\sin k_{i}} .
$$

From the boundary conditions $(22,23)$ we deduce that to obtain nontrivial solutions, the wavenumbers $k_{i}$ must verify $\cos k_{i}$ ch $k_{i}=-1$. Relations (24) and (25) are substituted into the governing equation (21), then the resulting equation is projected onto the free modes $\phi_{i}$. The complex eigenfrequencies $\omega$ are then determined from the solvability condition of the linear system obtained. Each eigenfrequency $\omega$ corresponds to a specific eigenmode of the instability made of a combination of the free modes $\phi_{i}$. The real part $\mathfrak{R e}[\omega]$ is the angular frequency $2 \pi f$ and its temporal growth rate is given by $-\mathfrak{I} \mathrm{m}[\omega]$. It follows that a negative value of $\mathfrak{I} \mathrm{m}[\omega]$ for at least one mode is indicative of a flapping instability of the filament. The computations are repeated with an increasing number $n$ of modes in (24) in order to ensure the convergence of the solution with a good enough accuracy. The theoretical results of Figs. 9 and 10 are obtained with $n=23$ resulting in angular frequencies $\mathfrak{R}[\omega]$ and growth rates $-\mathfrak{I m}[\omega]$ evaluated with an accuracy better than $0.2 \mathrm{~s}^{-1}$ for 
$l=35 \mathrm{~cm}$, and better than $0.1 \mathrm{~s}^{-1}$ for $l<20 \mathrm{~cm}$. For shorter filaments the convergence could be achieved with a decreasing number of modes (typically $n<10$ for $l<10 \mathrm{~cm}$ ).

The theoretical values for the critical velocity $U_{c}$ are plotted in Fig. 9 as a function of the filament length $l$ for the diameters $d=1.2 \mathrm{~mm}$ [Fig. 9(a)] and $1.8 \mathrm{~mm}$ [Fig. 9(b)]. A comparison with experimental results is also shown and reveals in both cases a good qualitative and quantitative agreement. It is noteworthy that this agreement is obtained without adjustable parameter. However, a slight shift of the calculated curves towards the lower length values can be noted. This may be due to the fact that the filament is considered as rectilinear on its whole length $l$ whereas the actual rectilinear part is shorter.

The instability mode destabilized at the threshold $U_{c}$ depends on the filament length $l$. Thus, the characteristic lobes of the theoretical curves $U_{c}(l)$ in Fig. 9 correspond to various instability modes that differ in their shapes and frequencies. Note that these instability modes are combinations of the Galerkin modes $\phi_{i}$ (and should not be confused with them).

Theoretical and experimental measurements of flapping frequencies are also compared in Fig. 9 for three filaments ( $l=12$ and $20 \mathrm{~cm}$ for $d=1.2 \mathrm{~mm}, l=12 \mathrm{~cm}$ for $d=1.8 \mathrm{~mm}$ ). In all cases, and although they are of the same order of magnitude, we note that the frequencies predicted by the model are systematically overestimated when compared to the experimental results. In the same manner, their dependence with the filament length, as it is observed during the experiments, is not fully recovered by the model. Nevertheless qualitative agreements can be found such as the dependence of the frequency $\omega$ on the free stream velocity $U$ which is predicted with a very good approximation. Also, when considering results for filaments of a given length $(l=12 \mathrm{~cm})$ but of different diameters $(d=1.2$ and $1.8 \mathrm{~mm})$ we can see that, in agreement with the experimental observations, the theory gives a flapping frequency $\omega$ that decreases as $d$ is increased.

\section{IV.DISCUSSIONS AND CONCLUSION}

\section{A. Effect of mass ratio}

Although the mass ratio $\mu$ has not been varied in the present experiments, a comparison with the recent study of Facchinetti et al. ${ }^{4}$ invalidates a VIV scenario to interpret our observations. These authors present 
indeed results on a freely hanging flexible cable of aspect ratio $l / d=250$, towed in a water tank with a mass ratio $\mu$ of about 2.07. They report the observations of waves propagating along the cable and consisting in traveling transverse (i.e. perpendicular to the free stream) displacements of the cable. These waves appear as soon as the Reynolds number $R e=U d / v$ exceeds the critical value of the appearance of the vortex-shedding (of about 50) and consequently are locked on these vortex shedding. These are evidences that these waves are excited by the periodic vortex shedding and a model of these VIV emphasises the resonance of the structure with the periodic fluid loading.

By contrast, for the same aspect ratio (a filament with $l=30 \mathrm{~cm}$ and $d=1.2 \mathrm{~mm}$ for instance, resulting in an aspect ratio $l / d$ of 250 ) we observe a critical value $R e_{c} \approx 610$ (see Fig. 2) with vertical displacements of the filament. This flapping frequency appears clearly not to be locked on the vortex shedding frequency that would correspond to a Strouhal number $S t=f d / U$ of 0.21 (see e.g. the recent review by Norberg ${ }^{12}$ ), while $S t$ is only of about $4 \times 10^{-3}$ for the flapping oscillations (considering the total excursion of the free end of the filament instead of the filament diameter as length scale, we obtain a Strouhal number of order $10^{-1}$ ); in this estimation, the incidence of the filament versus the flow has been taken into account with the cosine correction proposed by Williamson ${ }^{13}$. Therefore the flow-induced vibrations presented here appear to be clearly of a different nature that of the classical VIV. Our theoretical study shows that the flapping of the filaments results directly from a loss of stability of the filament stationary states under the action of external steady forces. Besides, this is also consistent with the VIV models (e.g. Facchinetti et al. ${ }^{4}$ ) that predict the narrowing of the synchronization domain when the mass ratio is increased. In our case, the VIV resonance domain is negligibly small and can not be invoked to explain our observations.

\section{B. Beyond the linear regime}

Finally we should also mention that our experiments show that the transition from a static state to flapping exhibits hysteresis and bistability. Actually, when experiments, as described in § II.B, are repeated with a decreasing velocity $U$, the flapping motion persists up to a value $U_{c \text { - }}$ lower than $U_{c}$. This is illustrated in Fig. 11 that shows the angular frequency of the flapping for increasing and decreasing $U$, for the filament $d=1.2 \mathrm{~mm}$ and $l=20 \mathrm{~cm}$. While flapping appears at a critical value $U_{c}$ of the wind velocity between 9.4 and 
$9.6 \mathrm{~m} \mathrm{~s}^{-1}$, it is maintained to $U_{c \text { - }}$ between 8.7 and $8.9 \mathrm{~m} \mathrm{~s}^{-1}$ when $U$ is decreased.

In the bistability domain $\left[U_{c-}, U_{c}\right]$, the filament can easily be forced to either a static state or flapping by external perturbations. For $U<U_{c-}$ and $U>U_{c}$ all attempts to change the spontaneous state of the filament are fruitless. Such an hysteretical cycle has also been observed in numerous flow-induced vibration problems as for in experiments on a filament in an axial flow (Païdoussis et al. ${ }^{5}$ ) or in a flowing soap film (Zhang et $\left.a l^{14}\right)$. This bistability is supposed to be responsible for the dispersion of the experimental critical velocities observed in Fig. 2.

\section{Conclusion}

Observations of flow-induced vibrations for a filament freely hanging in a vertical plane are reported. In contrast to low mass ratio situation, no VIV have been observed. The persistence of steady states up to Reynolds number of several hundreds, results in the appearance of another kind of instability where the filaments oscillate in the vertical plane. We have shown that this flapping results from a loss of stability of the filament static equilibrium states under the action of the steady fluid flow loads. Our theoretical results confirm the appearance of this instability and in particular the threshold values for its observation. The different characteristics of the flapping are also correctly predicted by the model and in particular the exchanges between different flapping modes when the length of the filaments is increased. Finally, a strong nonlinear behavior with the existence of a bistable regime has been detected experimentally. This would demand a nonlinear theoretical analysis in order to describe the saturation of flapping amplitude as $U$ is increased and the observed hysteretical behavior at threshold. 


\section{APPENDIX}

Expressions of the coefficients of equation (21):

$$
\begin{aligned}
& a=\frac{l^{2}}{E I} \rho_{f l} A U^{2} \cos ^{2} \theta, \\
& b=-\frac{l^{3}}{E I}\left[L+\left(\rho-\rho_{f l}\right) g A \sin \theta\right], \\
& c_{1}=-\frac{l^{3}}{E I}\left[\left(\rho-\rho_{f l}\right) g A \sin \theta+\frac{1}{2} \rho_{f l} d U^{2}\left(C_{D} \sin 2 \theta+6 R e^{-\frac{1}{2}} \cos \theta \sin ^{\frac{1}{2}} \theta\right)\right] \\
& c_{2}=-\frac{l^{3}}{E I} 2 \rho_{f l} A U \cos \theta, \\
& e_{1}=-\frac{l^{4}}{E I}\left(\rho+\rho_{f l}\right) A, \\
& e_{2}=\frac{l^{4}}{E I} \rho_{f l} d U\left[C_{D} \sin ^{2} \theta+3 R e^{-\frac{1}{2}} \sin ^{\frac{1}{2}} \theta\right] .
\end{aligned}
$$

\section{REFERENCES}

${ }^{1}$ R. D. Blevins, Flow-Induced Vibration (R.E. Krieger Publishing Company, Malabar, Florida, 1986).

${ }^{2}$ P. W. Bearman, "Vortex shedding from oscillating bluff bodies,” Ann. Rev. Fluid Mech. 16, 195 (1984).

${ }^{3}$ C. H. K. Williamson and R. Govardhan, "Vortex-induced vibrations," Ann. Rev. Fluid Mech. 36, 413 (2004).

${ }^{4}$ M. L. Facchinetti, E. de Langre, and F. Biolley, "Vortex-induced travelling waves along a cable,” Eur. J. Mech. B/Fluids 23, 199 (2004).

${ }^{5}$ M. P. Païdoussis, E. Grinevich, D. Adamovic, and C. Semler, "Linear and non-linear dynamics of cantilevered cylinders in axial flow. Part 1: Physical dynamics,” J. Fluids Struct. 16, 691 (2002). 
${ }^{6}$ J. L. Lopes, M. P. Païdoussis, and C. Semler, "Linear and non-linear dynamics of cantilevered cylinders in axial flow. Part 2: The equation of motion,” J. Fluids Struct. 16, 715 (2002).

${ }^{7}$ G. I. Taylor, “Analysis of the swimming of long and narrow animals,” Proc. R. Soc. A CCVIV, 158 (1952).

${ }^{8}$ M. J. Lighthill, “Note on the swimming of slender fish,” J. Fluid Mech. 9, 305 (1960).

${ }^{9}$ M. P. Païdoussis, “Dynamics of flexible slender cylinder in axial flow. Part 1. Theory,” J. Fluid Mech. 26, 717 (1966).

${ }^{10}$ M. P. Païdoussis, “Dynamics of cylindrical structures subjected to axial flow,” J. Sound Vib. 29, 365 (1973).

${ }^{11}$ C. Semler, J. L. Lopes, N. Augu, and M. P. Païdoussis, "Linear and non-linear dynamics of cantilevered cylinders in axial flow. Part 3: Nonlinear dynamics,” J. Fluids Struct. 16, 739 (2002).

${ }^{12}$ C. Norberg, "Fluctuating lift on a circular cylinder: review and new measurements,” J. Fluids Struct. 17, 57 (2003).

${ }^{13}$ C. H. K. Williamson, “Vortex dynamics in the cylinder wake,” Ann. Rev. Fluid Mech. 28, 477 (1996).

${ }^{14}$ J. Zhang, S. Childress, A. Libchaber, and M. Shelley, "Flexible filaments in a flowing soap film as a model for one-dimensional flags in a two-dimensional wind,” Nature 408, 835 (2000). 


\section{FIGURE CAPTIONS}

FIG. 1. Superimposed views of steady states of the filament $l=12 \mathrm{~cm}, d=1.2 \mathrm{~mm}$ for $U$ from 0 to $13.6 \mathrm{~m} \mathrm{~s}^{-1}$ $\left(U=0.0,1.1,2.2,3.4,4.5,6.0,7.5,9.5,11.5\right.$ and $13.6 \mathrm{~m} \mathrm{~s}^{-1}$, flow from right to left).

FIG. 2. Experimental thresholds $U_{c}$ for the appearance of flapping as function of the filament length $l$ for $d=1.2 \mathrm{~mm}(+)$ and $1.8 \mathrm{~mm}(\circ)$.

FIG. 3. Superimposed successive views of the flapping filament for $l=12 \mathrm{~cm}, d=1.2 \mathrm{~mm}, U=16.9 \mathrm{~m} \mathrm{~s}^{-1}$ (a) and $l=21 \mathrm{~cm}, d=1.8 \mathrm{~mm}, U=14.7 \mathrm{~m} \mathrm{~s}^{-1}$ (b). Flow from right to left.

FIG. 4. Flapping frequency $f$ as function of the free stream velocity $U$ for $l=12 \mathrm{~cm}(\bullet, \bigcirc), l=16 \mathrm{~cm}(\boldsymbol{\nabla}, \nabla)$, $l=20 \mathrm{~cm}(\square, \boldsymbol{\varpi}), l=24 \mathrm{~cm}(\diamond, \diamond)$. Open and filled symbols are for $d=1.2 \mathrm{~mm}$ and $d=1.8 \mathrm{~mm}$, respectively.

FIG. 5. Forces and moment acting on a steady filament element ds.

FIG. 6. Theoretical static equilibrium shapes of the filament calculated for $C_{D}=0.8$ (solid line) and $C_{D}=0.9$ (dashed line). Same conditions as experiments of Fig. 1.

FIG. 7. Experimental (+) and theoretical (with $C_{D}=0.8$ (solid line) and $C_{D}=0.9$ (dashed line)) values of the incidence angle at the free end $\theta(s=0)$, for the filament $l=12 \mathrm{~cm}$ and $d=1.2 \mathrm{~mm}$, as function of the free stream velocity $U$.

FIG. 8. Scheme of a filament element ds undergoing a small displacement $y(s, t)$ from its static equilibrium position (dashed line).

FIG. 9. Experimental (+) and theoretical (Galerkin approximation with $C_{D}=0.8$ (solid line) and $C_{D}=0.9$ (dashed line)) values of the critical velocity $U_{c}$ as function of the length $l$ for the filament of diameter $d=1.2 \mathrm{~mm}(\mathrm{a})$ and $d=1.8 \mathrm{~mm}(\mathrm{~b})$. 
FIG. 10. Comparison of experimental and theoretical values of the flapping frequency $\omega$. Experiments: $l=12 \mathrm{~cm}$ and $d=1.2 \mathrm{~mm}((), l=20 \mathrm{~cm}$ and $d=1.2 \mathrm{~mm}(\square), l=12 \mathrm{~cm}$ and $d=1.8 \mathrm{~mm}(\bullet)$. Theory: $l=12 \mathrm{~cm}, d=1.2 \mathrm{~mm}, C_{D}=0.8$ (solid line) and $C_{D}=0.9$ (dashed line), $l=20 \mathrm{~cm}, d=1.2 \mathrm{~mm}, C_{D}=0.8$ (dotted line), $l=12 \mathrm{~cm}, d=1.8 \mathrm{~mm}, C_{D}=0.8$ (dash-dot line).

FIG. 11. Flapping frequency $\omega$ for increasing $(\triangle)$ and decreasing $(\boldsymbol{\nabla})$ free stream velocity $U(l=20 \mathrm{~cm}$, $d=1.2 \mathrm{~mm})$ 


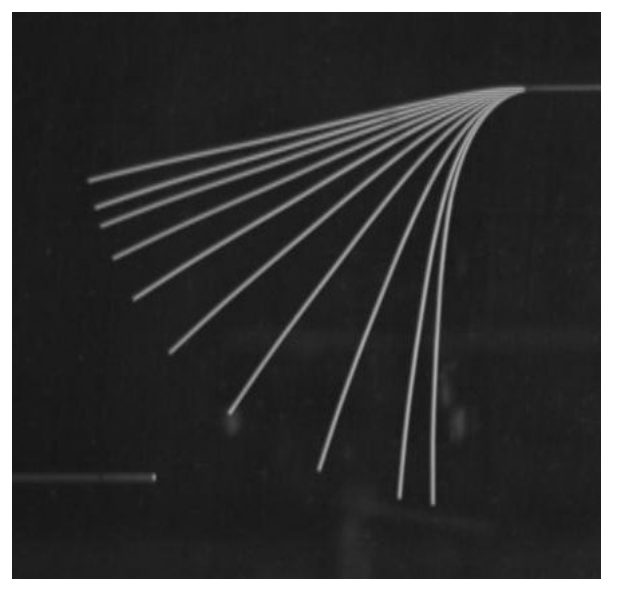

FIG. 1. 


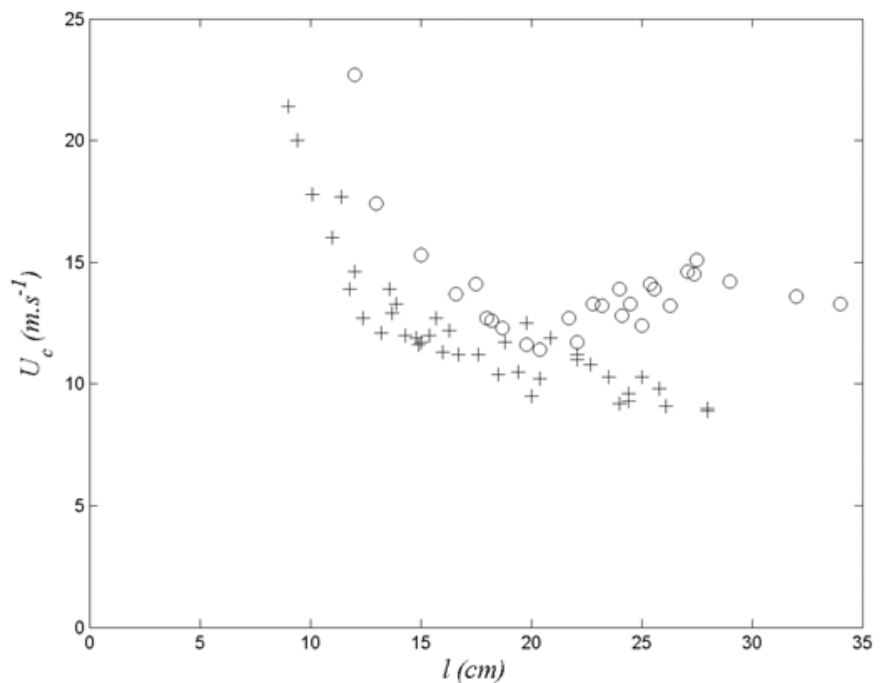

FIG. 2. 


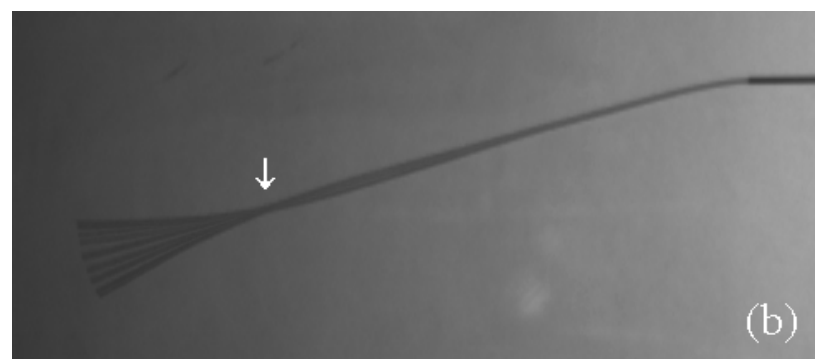

FIG. 3. 


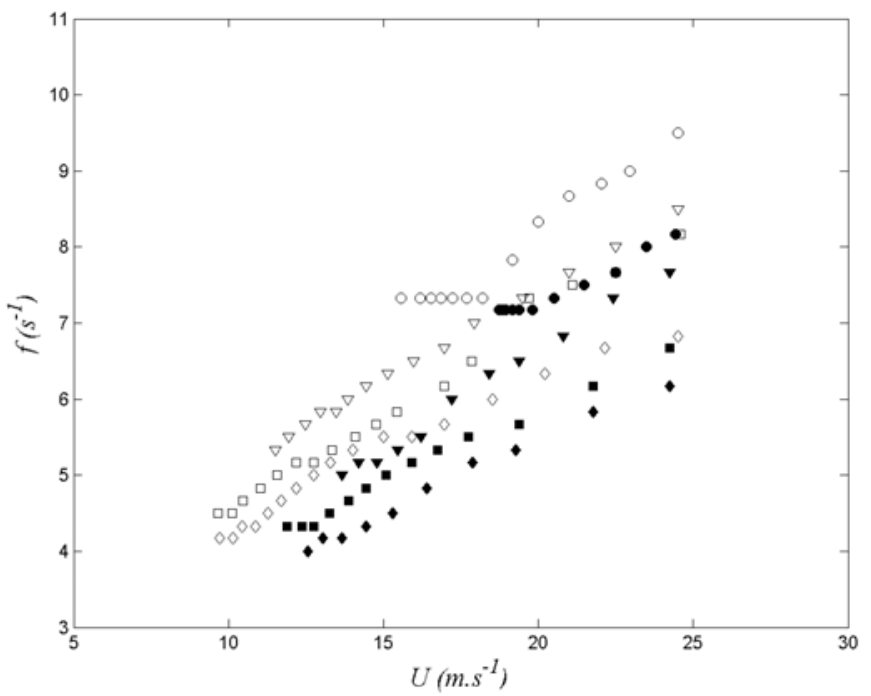

FIG. 4. 


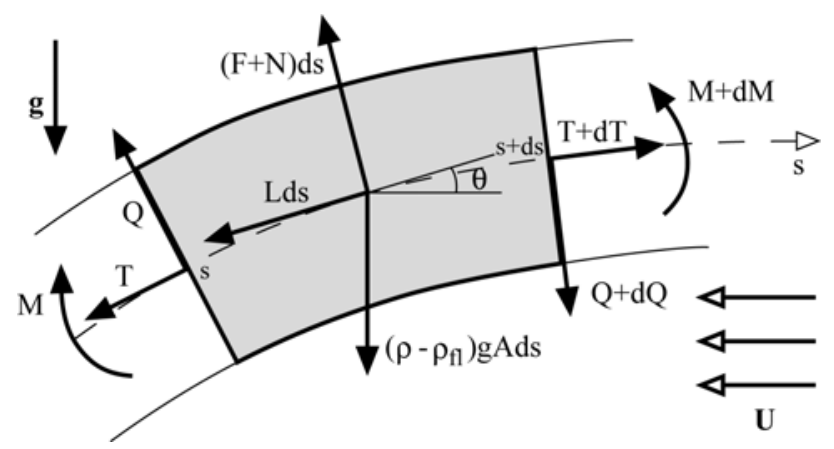

FIG. 5. 


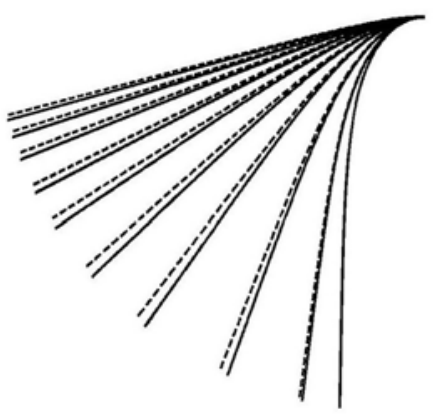

FIG. 6. 


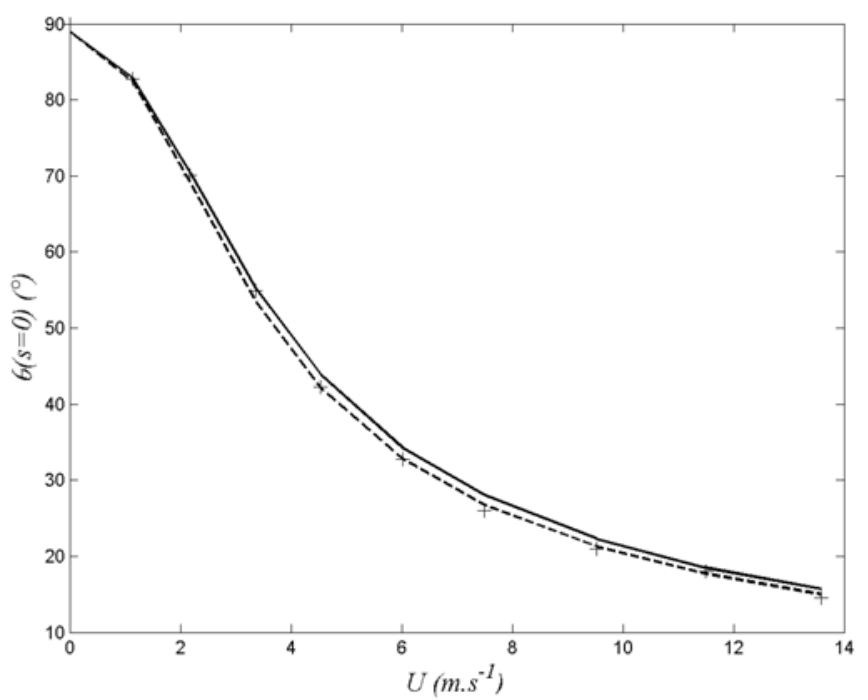

FIG. 7 


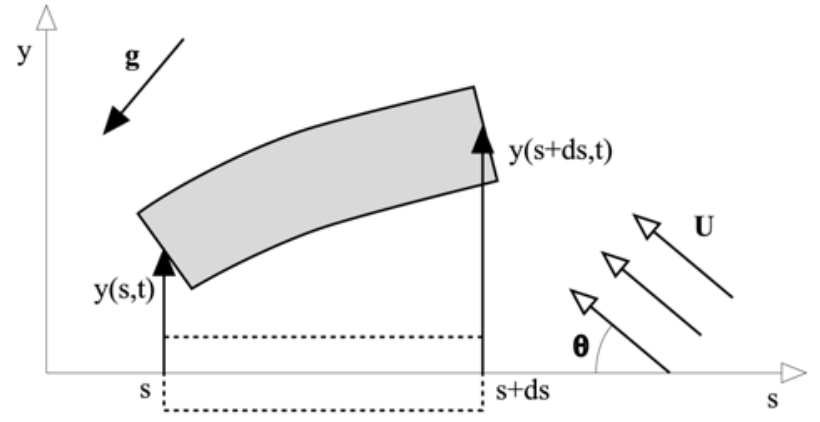

FIG. 8. 

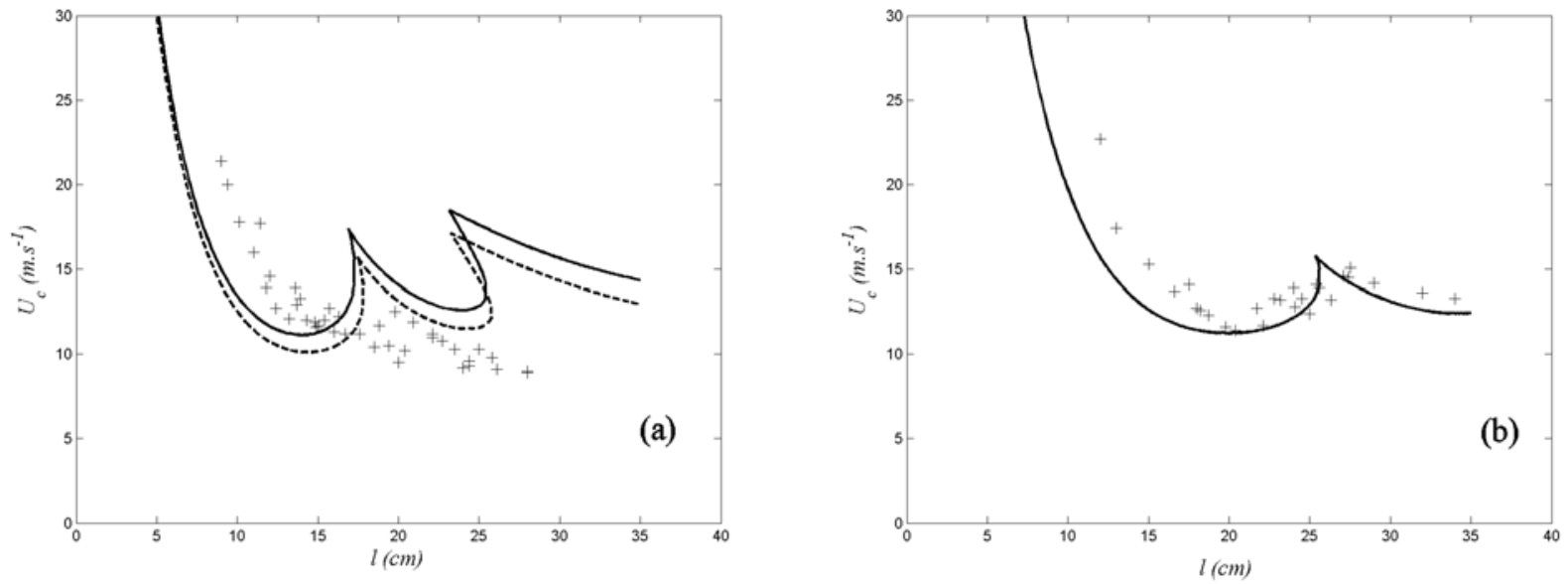

FIG. 9. 


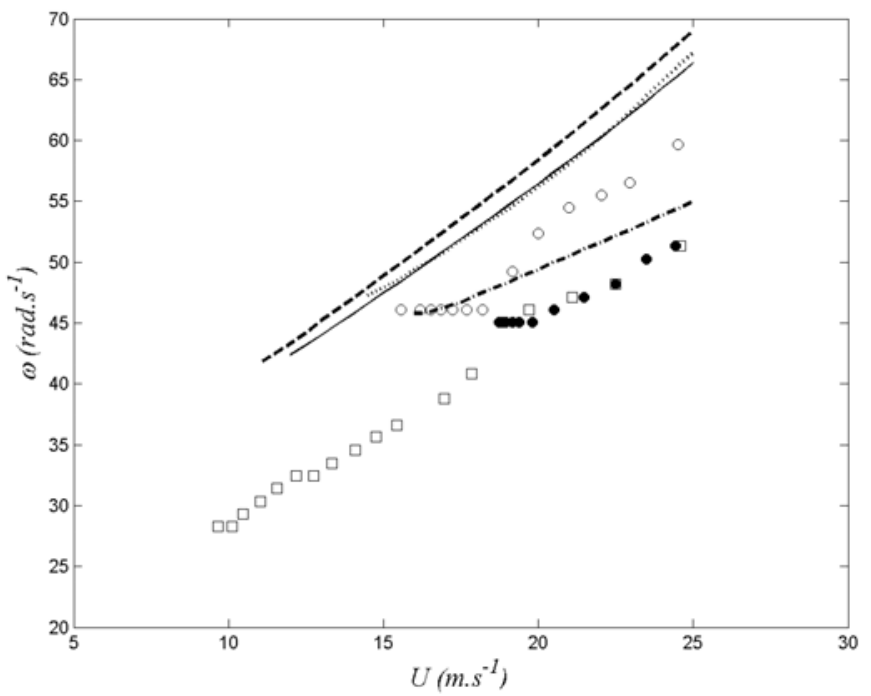

FIG. 10 


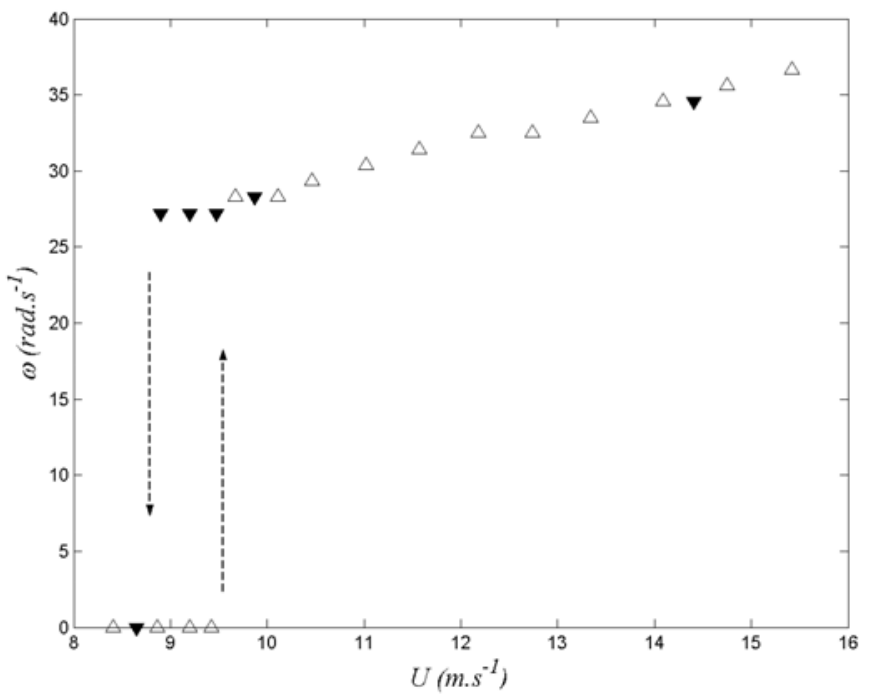

FIG. 11. 